\title{
Locked Posterior Dislocation of Shoulder With Fracture of the Lesser Tuberosity of the Humerus: A Case Report and Review of the Literature
}

\author{
Ali Tabrizi,${ }^{1,}{ }^{*}$ Mir Bahram Safari, ${ }^{1}$ Hassan Taleb, ${ }^{1}$ and Nasrin Navaeifar ${ }^{1}$ \\ ${ }^{1}$ Assistant Professor of Orthopedics Surgery Department, Imam Khomeini Hospital, Urmia University of Medical Sciences, Urmia, IR Iran \\ "Corresponding author: Ali Tabrizi, MD, Moderres Ave, Imam Khomeini Educational Hospital, Urmia University of Medical Sciences, Urmia, IR Iran. Tel: +98-9143130829, E-mail: \\ Dr.tabrizi.ali@hotmail.com
}

Received 2016 May 05; Revised 2016 July 22; Accepted 2016 October 01.

\begin{abstract}
Introduction: Posterior shoulder dislocation associated with fracture of the lesser tuberosity is considered as a rare shoulder trauma. It can lead to the locked shoulder with a very high possibility of unsuccessful closed reduction.

Case Presentation: In this case, the patient was a 30-year-old male motorcyclist with the right shoulder injury who had posterior shoulder dislocation with lesser tuberosity fracture. Early attempt for closed reduction was unsuccessful and led to iatrogenic brachial plexus injury. This patient is treated by open reduction and fracture fixation with screw. Subscapularis partial rupture was repaired. Finally, full stability was achieved.

Conclusions: Fracture fixation plays a crucial role in stability after reduction. Several techniques have been suggested for surgical treatment including fixation with screw and the modified McLaughlin technique. Similar to the previous case reports, according to our experience, careful attention to the fracture concomitant with posterior dislocation is of the utmost importance.
\end{abstract}

Keywords: Posterior Fracture Dislocation, Lesser Tuberosity Fracture, Reverse Hill-Sachs Lesion

\section{Introduction}

Posterior fracture-dislocation of the shoulder is one of the rare injuries, which is often due to the seizure and high-energy trauma. Generally, it is accounted for about $2 \%$ of the shoulder dislocations (1). It is strongly possible that this type of injury be missed (2). Meanwhile, fracture of the lesser tuberosity of the humerus can be associated with posterior shoulder dislocation (3). According to the McLaughlin study, 22 (3.8\%) out of 581 cases of shoulder dislocations had posterior shoulder dislocation. In more cases, the impression fracture in the humeral head is possibly reverse Hill-Sachs lesion and it is often covered less than $25 \%$ of the articular surface (4). In some very rare cases, fracture of lesser tuberosity is associated with the locked posterior dislocation, in which there is no possibility of the closed reduction (5). Therefore, the diagnosis of simultaneous dislocation and fracture is essential to decide for the appropriate therapy. In this report, a patient with a simultaneous posterior fracture-dislocation of the shoulder is described that closed reduction was not possible in the emergency department and open reduction and fixation of fracture were needed, as well.

\section{Case Presentation}

The patient was a 30-year-old male motorcyclist with the right shoulder injury due to the reversal of the bicycle admitted to the emergency department of our hospital. He was complaining of the shoulder pain and the limited motion in the shoulder, as well. No injury and trauma were observed in other parts of his body. In the physical examination, there was a severe tenderness in the right shoulder. No passive and active motions of the shoulder were observed. The neurological and vascular examinations were normal with no problems.

Shoulder radiography in the emergency department showed a fracture of the humeral head associated with the posterior dislocation (Figure 1). There was no associated fracture in the elbow and wrist radiographs. In the emergency department, closed reduction under sedation was performed due to the presence of posterior dislocation, which was not successful and it was consulted with the orthopedic department of the center. Three-dimensional CT scan was ordered after re-examination of the patient, since there was a fracture of the lesser tuberosity associated with the locked posterior dislocation (Figures 2 and 3).

In the re-examination of the patient, there was a nerve injury in the brachial plexus as a complete functional disorder in the radial nerve, but the median and ulnar nerves were normal. The patient was subsequently nominated for open surgery. General anesthesia was applied for the patient. Deltopectoral approach was used for the surgery. Biceps tenotomy was performed first and lesser tuberosity fragment was taken with the nonabsorbable PDS suture. 


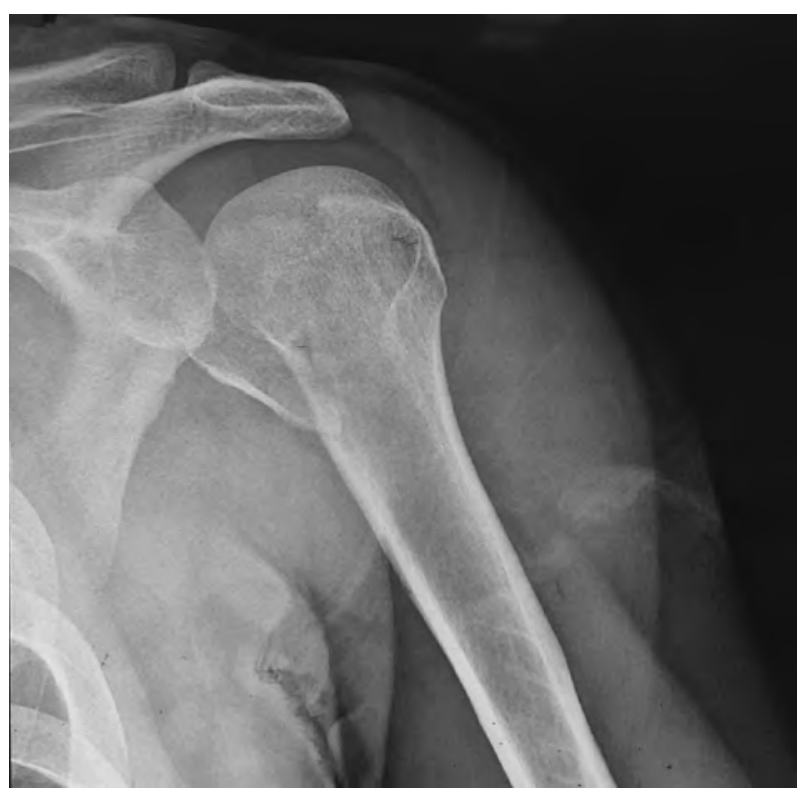

Figure 1. Radiography of the Patients' Shoulder Indicating Posterior Dislocation Associated With Fracture of the Lesser Tuberosity

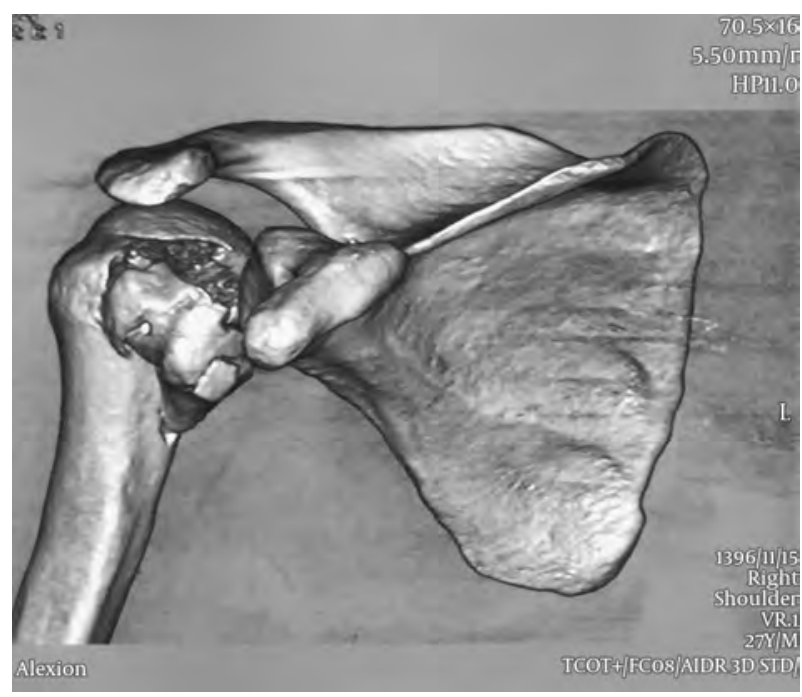

Figure 2. Three-Dimensional CT Scan View Showing the Locked Fracture-Dislocation of the Posterior Shoulder

Open reduction was done using a bone lever and pushing straight forward from the shoulder. After reduction before fixation, the full stability was achieved in external rotation in the passive movements, but there was instability in full internal rotation that a pin (No. 2) from acromion to the humeral head was used temporary (Figure 4). After open reduction of the humeral head, fixation of the fractured

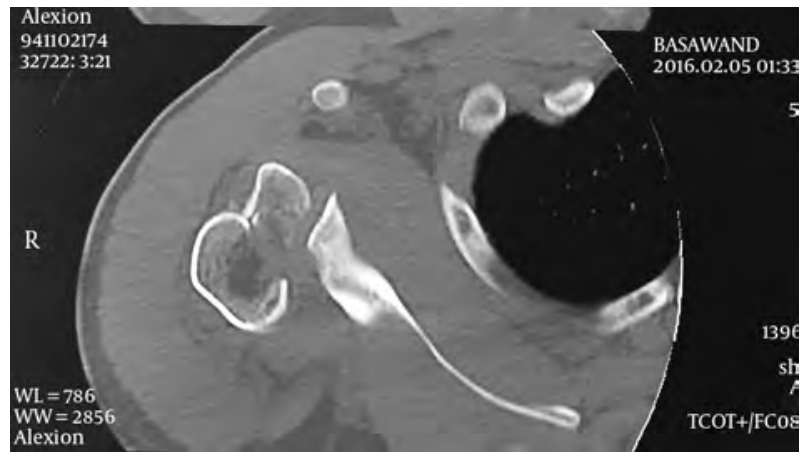

Figure 3. Axial Section of CT Scan View Showing the Locked Fracture-Dislocation of the Posterior Shoulder

fragment with the partial thread screw(No 4.5) was needed due to the fracture of lesser tuberosity and unstable reattached shoulder and a countersunk screw was used. Subscapularis muscle was torn that was repaired (Figure 5). Because the Herbert screws did not have the appropriate size to insert to the articular humeral surface, we use screws number 4.5. We tried to exactly countersink to avoid causing the secondary impingement in operation time; there was no impingement in the shoulder passive range of motion. Subscapularis partial rupture was repaired, it can cause partial tear of the subscapularis because of the nature of the fracture dislocation and locking the glenoid rim happened. Finally, the shoulder was fixed by a special brace in adduction and internal rotation positions. Two weeks after the surgery, trans-acromial pin was removed and rehabilitation was started. First, muscle strengthening and pendulum motion exercises were performed for a month. There has been still the radial nerve injury after a month. The axonal injury was observed in the electromyography that there was a possibility of traction injury. The observed iatrogenic neurapraxia was due to the attempt for initial reduction in the emergency department, which was continued conservatively. In the follow-up period after three months, shoulder pain was completely disappeared. Range of shoulder motion was obtained 150 degrees for forward flexion, 80 degrees for the external rotation, and internal rotation to the level of the tenth rib to the thoracic vertebra.

\section{Discussion}

Posterior dislocation of the shoulder was for the first time introduced by cooper in 1839 in an epileptic patient. The concurrency of posterior dislocation and fracture is rare (6). Overall, in the Neer report, in about $0.9 \%$ of 


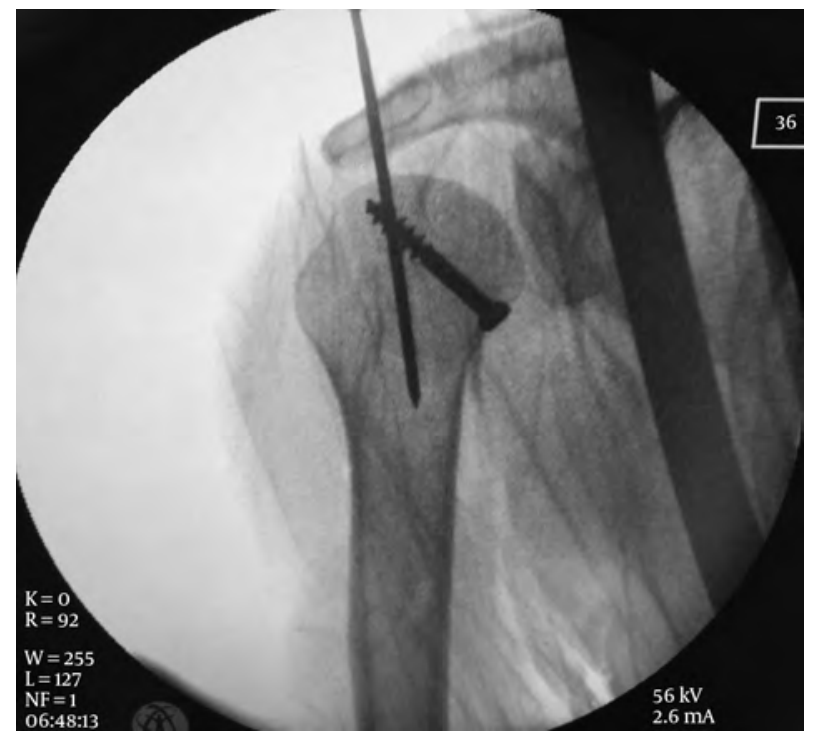

Figure 4. Intraoperative Fluoroscopy View After Open Reduction and Fixation Using Screw in the Lesser Tuberosity

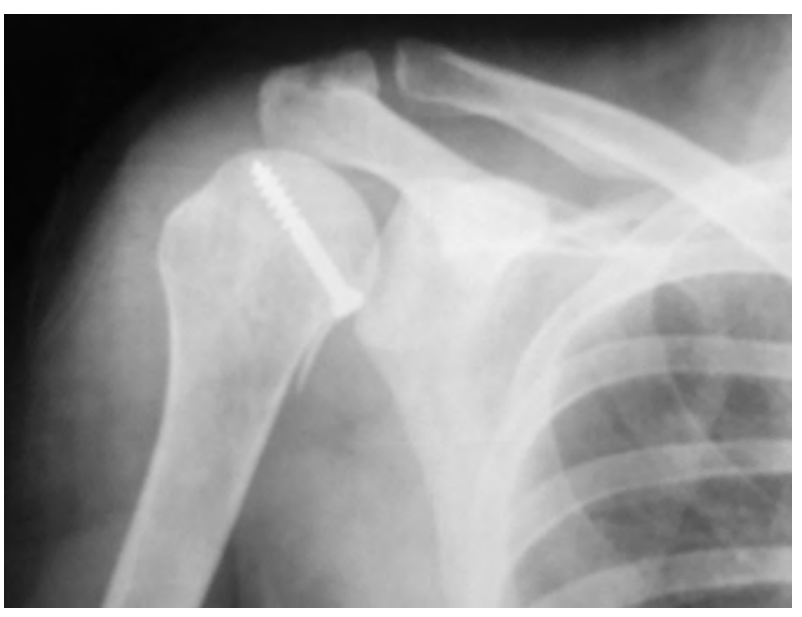

Figure 5. The Ultimate Radiography of the Patients Two Weeks After the Surgery Indicating the Full Stability in the Shoulder Joint

1500 cases, posterior dislocation was associated with fractures. Impression fracture in the humeral head is defined as locked posterior dislocation of the shoulder (7). The locked dislocations are considered as a determining key factor for unsuccessful closed reduction. Diagnosis of this condition is often delayed or missed (1). The prompt diagnosis and treatment of fracture-dislocation is crucial. Avascular necrosis and collapse are the two most possible important complications. On the other hand, the fracture of lesser tuberosity is often occurred simultaneously with posterior dislocation (8). The locked posterior dislocation is accounted for about one-third of posterior dislocations that strongly can change the therapy and prognosis (6). In the Benhamida study, one close reduction out of three locked posterior dislocation was failed and open reduction and using a special technique for stability were needed in all three cases (9). In this report, open reduction, subscapularis transfer or the McLaughlin technique were used. Satisfactory results were obtained in the two cases; in one case, dislocation re-occurred after three days; and posttraumatic osteoarthritis was observed in one patient (9). Moussa et al. have been reported a patient with an associated locked posterior dislocation with the fracture anterior impression and the acute tears of subscapularis tendon. The McLuaghlin technique was used due to the late diagnosis and the desired results were achieved (10). In the Liu et al. study (2014), several cases of twopart lesser tuberosity associated with posterior dislocation have been examined. The great results have been obtained from the fracture of lesser tuberosity surgery in the locked posterior dislocations (11). The modified McLaughlin technique has been used in 18 cases. The mean length from injury to the surgery was 40 days (11). In the Tey study (2007) in Singapore, posterior fracture-dislocation of the shoulder has treated without using metallic implants (12). The good results have obtained from a 34-year-old patient with posterior fracture-dislocation for whom the deltopectoral shoulder approach and open reduction have been used and nonabsorbable polyester has been used for lesser tuberosity fixation (12). The accurate understanding of the locked posterior dislocation is crucial, since in such cases, the reduction can be unsuccessful (6). In the studied patient, the effort to close reduction in the emergency department was not successful due to the rarity, lack of sufficient accuracy and the diagnosis. Sedation in the emergency department for close reduction and the patient without enough muscle relaxation can be dangerous increasing the possibility of iatrogenic injury. High traction can lead to the nerve injury in the brachial plexus. For each patient, the recovery rate is different depending on the site and type of the injury. In the Ben-David study, $50 \%$ of the 22 cases have been improved through a year (13). It seems that using additional imaging procedures can be helpful in the posterior fracture-dislocation for the accurate diagnosis of the locked fracture-dislocation cases (12). According to Richards RR reports, a brachial plexus injury occurs after shoulder instability treatment, especially after a Putti-Platt or a Bristow procedure and the lesion does not rapidly, progressively, and completely recover, the brachial plexus should be explored since there is a high likelihood of structural neurologic injury (14). There are very few publications describe the iatrogenic paralysis and management. 
Closed reduction under general anesthesia is considered as a selective therapy for posterior dislocation of the shoulder and the joint stability can be achieved via reduction in cases with the defects in the humeral head leading to impression fracture of less than $25 \%$ of the articular surface (1). Open reduction and fixation are required when the defects in the humeral head articular surface are greater than $25 \%$ (1). The tight locking in the large impression fracture is regarded as the most important factor for unsuccessful closed reduction in the posterior dislocation (1). The simultaneous fracture at the anatomical neck, soft tissue damage in the infraspinatus tendon, biceps tendon interposition, and dislocation over three weeks are considered as the other factors involved in the late diagnosis (1). In the studied patient, stability in the shoulder joint achieved due to the fixation of the fracture of lesser tuberosity using screw and subscapularis tendon repair.

\section{Acknowledgments}

This study was supported by Urmia University of Medical Sciences.

\section{Footnote}

Conflict of Interests: The authors declared that there was no conflict of interest.

\section{References}

1. Cicak N. Posterior dislocation of the shoulder. J Bone Joint Surg Br. 2004;86(3):324-32. [PubMed: 15125117]

2. Cuffolo G, Coomber R, Burtt S, Gray J. Posterior shoulder dislocation while lifting weights: a missed diagnosis. BMJ Case Rep. 2014;2014 doi: 10.1136/bcr-2013-202156. [PubMed: 24557475].
3. Gruson KI, Ruchelsman DE, Tejwani NC. Isolated tuberosity fractures of the proximal humeral: current concepts. Injury. 2008;39(3):284-98. doi: 10.1016/j.injury.2007.09.022. [PubMed: 18243203].

4. Mc LH. Posterior dislocation of the shoulder. J Bone Joint Surg Am. 1952;24 A(3):584-90. [PubMed: 14946209].

5. Hawkins RJ, Neer CS 2nd, Pianta RM, Mendoza FX. Locked posterior dislocation of the shoulder. J Bone Joint Surg Am. 1987;69(1):9-18 [PubMed: 3805075].

6. Mimura T, Mori K, Matsusue Y, Tanaka N, Nishi Y, Kobayashi M. Closed reduction for traumatic posterior dislocation of the shoulder using the 'lever principle': two case reports and a review of the literature. $J$ Orthop Surg (Hong Kong). 2006;14(3):336-9. [PubMed: 17200541]

7. Neer CS 2nd. Displaced proximal humeral fractures. I. Classification and evaluation. J Bone Joint Surg Am. 1970;52(6):1077-89. [PubMed: 5455339].

8. Gerber C, Schneeberger AG, Vinh TS. The arterial vascularization of the humeral head. An anatomical study. J Bone Joint Surg Am. 1990;72(10):1486-94. [PubMed: 2254356].

9. Benhamida MK, Ouertatani M, Hasayri I, Benhassine A, Meherzi $\mathrm{M}$, Bouhdiba S, et al. Locked posterior dislocation of the shoulder: A report of three cases. Chir Main. 2015;34(2):98-101. doi: 10.1016/j.main.2014.12.006. [PubMed: 25765116].

10. Moussa ME, Boykin RE, Earp BE. Missed locked posterior shoulder dislocation with a reverse Hill-Sachs lesion and subscapularis rupture. Am J Orthop (Belle Mead NJ). 2013;42(12):E121-4. [PubMed: 24471154].

11. Liu X, Zhu Y, Lu Y, Li F, Wu G, Jiang C. Locked Posterior Shoulder Dislocation Associated With Isolated Fractures of the Lesser Tuberosity: A Clinical Study of 22 Cases With a Minimum of 2-Year Follow-up. J Orthop Trauma. 2015;29(6):271-5. doi:10.1097/BOT.0000000000000276. [PubMed: 25470563]

12. Tey IK, Tan AH. Posterior fracture-dislocation of the humeral head treated without the use of metallic implants. Singapore Med J 2007;48(4):e114-8. [PubMed: 17384866].

13. Ben-David B, Stahl S. Prognosis of intraoperative brachial plexus injury: a review of 22 cases. Br J Anaesth. 1997;79(4):440-5. [PubMed: 9389259].

14. Richards RR, Hudson AR, Bertoia JT, Urbaniak JR, Waddell JP. Injury to the brachial plexus during Putti-Platt and Bristow procedures. A report of eight cases. Am J Sports Med. 1987;15(4):374-80. [PubMed: 3661820]. 